Research Article

\title{
Time to First-Line Antiretroviral Treatment Failure and Its Predictors among HIV-Positive Children in Shashemene Town Health Facilities, Oromia Region, Ethiopia, 2019
}

\author{
Endale Zenebe, ${ }^{1}$ Assefa Washo $\left(\mathbb{1}^{2},{ }^{2}\right.$ and Abreham Addis Gesese $\mathbb{( i}^{1}$ \\ ${ }^{1}$ Jimma University, College of Public Health and Medical Science, Department of Epidemiology, Jimma, Ethiopia \\ ${ }^{2}$ Hawassa College of Health Science, Department of Public Health, Hawassa, Ethiopia \\ Correspondence should be addressed to Assefa Washo; washassefa@gmail.com
}

Received 8 September 2020; Revised 30 June 2021; Accepted 9 July 2021; Published 18 August 2021

Academic Editor: Jacek Karwowski

Copyright ( $\odot 2021$ Endale Zenebe et al. This is an open access article distributed under the Creative Commons Attribution License, which permits unrestricted use, distribution, and reproduction in any medium, provided the original work is properly cited.

\begin{abstract}
With expanding pediatric antiretroviral therapy access, children will begin to experience treatment failure and require second-line therapy. In resource-limited settings, treatment failure is often diagnosed based on the clinical or immunological criteria which occur way after the occurrence of virological failure. Previous limited studies have evaluated immunological and clinical failure without considering virological failure in Ethiopia. The aim of this study was to investigate time to first-line antiretroviral treatment failure and its predictors in Shashamene town health facilities with a focus on virological criteria. Methods. A retrospective cohort study was conducted in three health facilities of Shashamene town, Oromia Regional State, from March 1 to 26, 2019. Children aged less than 15 years living with HIV/AIDS that were enrolled on ART between January 1, 2011, and December 30, 2015, in Shashamene town health facilities were the study population. Data were extracted using a checklist, entered into EpiData version 3.1, and exported to SPSS version 20 for data analysis. Cox proportional hazard regression was used to determine the predictors of time to first-line treatment failure. Result. The median survival time to virological failure was 30 months with IQR of 24.42 to 44.25. Baseline WHO stages 3 and 4 with AHR $=5.69$ (95\% CI: 2.07-15.66) and NVP-based NNRT at initial treatment with $\mathrm{AHR}=2.72(1.13-6.54)$ were the independent predictors of time to treatment failure. Conclusion. The median survival time of first-line antiretroviral treatment failure was moderate in the study area as compared to other studies. The incidence density of treatment failure in this study was low as compared to other studies. The finding also demonstrated that children treated with nevirapine-based nonnucleoside reverse transcriptase inhibitors at initial and advanced WHO clinical stages at baseline were at higher risk of treatment failure.
\end{abstract}

\section{Introduction}

Worldwide, AIDS now accounts for 3\% of deaths in children under five years of age- $6 \%$ of those in sub-Saharan Africa, where AIDS has become one of the major killers of young children. Without HIV care, including antiretroviral therapy, the progression of HIV infection in children is particularly aggressive [1]. Sub-Saharan Africa has the largest burden of pediatric HIV in the world. A global target has been set for the eradication of pediatric HIV by 2015, but there are still so many complex issues faced by HIV-infected and affected children in the subcontinent [2].
The burden of morbidity and mortality associated with HIV infection has decreased over the past decade as access to ART has increased [3]. After more than ten years of use, highly active antiretroviral therapy (HAART) treatment's effect has been documented in all WHO European region countries reporting increased survival, decreased HIV-associated mortality, and vastly improved quality of life [4].

Delays detecting treatment failure and switching to second-line combination antiretroviral therapy (cART) are often observed in human immunodeficiency virus- (HIV-) infected children in low-middle-income countries (LMIC). Reported regimen switching rates have been lower than 
expected. The absence of virological monitoring is associated with delayed switching and consequent accumulation of resistance mutations to nucleoside reverse transcriptase inhibitors (NRTIs) $[5,6]$.

Studies in LMIC have reported high rates of virological suppression in children up to 5-6 years after treatment initiation. However, treatment failure rates of $10-34 \%$ were observed among children after 2-3 years of cART [6]. From the study done in Uganda and Mozambique, the median time to treatment failure was 379 days (IQR: 229-649) [6].

In Ethiopia, based on the 2014 estimate, 178,500 children under 15 years need ART. In 2016, there were an estimated 748,933 people living with HIV including 78,834 children, and the total estimated new HIV infections were 21,565 and among them children under 15 were 2,212. In 2015, of the total number of people who needs ART, i.e., 636,556 people, 391,844 (61.6\%) accessed antiretroviral treatments; however, the number of children under 15 was only 22,967 (27.3\%) [7].

The Ethiopian Pediatric HIV Cohort (EPHIC) was established to identify clinical and laboratory predictors of virological treatment failure to ultimately develop a clinicalimmunological prediction rule with area under the curve of $>0.80$ for detecting first-line antiretroviral therapy failure (ARTF). It will also assess the performance of the current WHO guidelines for the detection of first-line ARTF in children. Many studies have reported the success of highly active antiretroviral therapy in improving clinical and immunological outcomes of children. However, as the use of HAART increases, the issue of drug resistance and subsequent treatment failure presenting as one or more of clinical, immunological, or virological ART failure appears as a challenge $[8,9]$.

Antiretroviral drugs are medications to be taken for life and they need follow-up to make sure they are still helping to achieve maximal viral suppression so that in the event they failed to do so, the regimen could be changed immediately [9]. Optimization of the clinical management of PLWH and prompt diagnosis of treatment failure are becoming increasingly critical in the context of lifelong treatment and limited drug availability [6].

The diagnosis of treatment failure is guided by viral load testing in high-income countries; however, this is not the case in lowest income countries. This is because of the sophisticated and expensive laboratory facility and training of personnel needed for determining viral load. As a result, in resource-limited settings, treatment failure is often diagnosed based on the clinical or immunological criteria which occur way after the occurrence of virological failure. Keeping patients on a failing regimen leads to the reversal of clinical conditions of patients to the pretreatment state and development of drug resistant strains. Once drug-resistant virus starts transmitting in the population, the consequences will be devastating. ART failure is not a common diagnosis in most centers in Ethiopia. Very few patients among the needy started on second-line ART regimens [10, 11]. Previous limited studies have evaluated immunological and clinical failure without considering virological failure in Ethiopia. This study, therefore, aims to determine time to first-line treatment failure and its predictors in Shashamene town health facilities with a focus on virological criteria, which is a gold standard for monitoring ART.

Knowing predictors that can help to predict treatment failure will help to identify those clients that are at a higher risk of failure. Armed with this information, clinicians could give such patients special attention during their follow-up and the limited resources available for diagnosing treatment failure can be used for them.

\section{Methods and Materials}

2.1. Study Area and Period. The study was conducted in Shashamene town, Oromia Regional State. Shashamene is a capital town of West Arsi Zone, Oromia Region, Ethiopia. The town lies on the Trans-African Highway 4 Cairo-Cape Town, about 150 miles $(240 \mathrm{~km})$ from the capital of Addis Ababa. The town has a total population of 264,780 in 2017, of which $133566(50.4 \%)$ are male, 131,214 (49.6\%) are female, and 43,424 are children under 15 years of age. 484 children were enrolled on ART from January 1, 2011, to December 30, 2015. The town has 8 kebeles with two public hospitals and four public health centers. Of these health facilities, one health center and two hospitals were provided ART service for the last seven years. The study was conducted from March 1 to 26, 2019.

2.2. Study Design. Facility-based retrospective cohort study was conducted.

2.2.1. Population. All children living with HIV/AIDS, aged less than 15 years, and on ART at Shashamene public health facilities were the source population while HIV-positive children that were enrolled on ART between January 1, 2011, and December 30, 2015, are the study population. Children transferred into ART with baseline information were included in the study and children whose intake form was not completed (data that have no commencement date of ART initiation and patient age) were excluded from the study.

2.3. Sample Size Determination. The total number of cases from January 1, 2011, to December 30, 2015, from three facilities was 484 . The power of the study was calculated by using STATA version 12. The exposure status for PMTCT and using nevirapine group regime was considered and the most significant predictor of first-line antiretroviral treatment failure, nevirapine group regime, was used, which was taken from the study conducted on predictors of virological failure in South Africa; the probability of event (probability of virological failure) in this study is $0.193, \alpha=0.05$, and AHR for nevirapine group regime is 1.8 [12]. Accordingly, the calculated power of the study was $81.1 \%$. 


\subsection{Study Variables}

2.4.1. Dependent Variables. The outcome variable was survival time or time to first-line treatment failure of HIVinfected children after starting HAART. The survival time was measured using months from the time of HAART initiation until the time to first-line treatment failure. Survival times of children who were still alive as of February 30,2018 , on first-line treatment, lost to follow-up, and dead were considered censored times.

2.4.2. Independent Variables. Independent variables are given as follows:

Sociodemographic characteristics: age, sex, child's primary caregiver, and nutritional history

Baseline clinical factors: WHO clinical stages, nutritional status, opportunistic illness (OI), and TB infection

Baseline immunological information: immunization status, CD4 count, and hemoglobin level

Treatment-related factors: the last drug adherence, type of initial regimens, prophylaxis use, and maternal exposure status to PMTCT

2.5. Variable Definition. The definitions of variables are given as follows:

Baseline measurements were those taken closest to ART initiation and within 6 months (CD4 and viral load) or 2 weeks (weight) prior to 1 week after the commencement of ART.

CD4 count categorized as per the WHO is the appropriate classification to describe their immunological level. Children under age 1 and who had CD4 cell count $<1500$ cells $/ \mathrm{mm}^{3}$; children aged between 1 and 3 years and who had CD4 cell count $<750$ cells $/ \mathrm{mm}^{3}$; children aged between 3 and 5 years and who had CD 4 cell count $<350$ cells $/ \mathrm{mm}^{3}$; and children aged between 5 and 15 years and had CD 4 cell count $<200$ cells $/ \mathrm{mm}^{3}$ will be categorized as having CD4 cell count below threshold [13].

Hemoglobin level was retrieved from record to assess anemia; for age 6-59 months, <11 g/dl; for 5 to 12 years, $<11.5 \mathrm{~g} / \mathrm{dl}$; and for 12 to 15 years, $<12 \mathrm{~g} / \mathrm{dl}$ was categorized as anemic otherwise not anemic [14].

Adherence to HAART was measured by the last adherence level recorded on the follow-up form and classified as good $>95 \%$, fair $85-94 \%$, and poor $<85 \%$ based on the percentage of drug dosage calculated from the total monthly doses of HAART drugs.

Nutritional status was categorized as undernutrition for under-five children if the weight for age of a child is below (-2SD) the standard deviation score, the standard WHO weight for age z-scores. Body mass index (BMI) was calculated for children 5 years old or above, and BMI less than $16 \mathrm{~kg} / \mathrm{m}^{2}$ was categorized as undernutrition [1].

Clinical failure is a new or recurrent clinical event indicating advanced or severe immune defiance (WHO clinical stage 3 and 4 clinical condition with exception of TB) after 6 months of effective treatment [15].

Immunological failure: children younger than 5 yearsexhibit persistent CD4 levels below 200 cells $/ \mathrm{mm}^{3}$, and those older than 5 years exhibit persistent CD4 levels below 100 cells $/ \mathrm{mm}^{3}$ [15].

Virological failure is defined as viral load above 1000 copies/mL based on two consecutive viral load measurements in 3 months with adherence support following the first viral load test. An individual must be taking ART for at least 6 months before it can be determined that the regimen has failed [15].

First-line antiretroviral treatment failure: in this study, treatment failure was considered when, due to virological failure, first-line regimen is changed to secondline regimen.

2.6. Data Collection Technique and Procedure. Three health facilities were selected based on the duration of the ART service. Data extraction tools were adapted from the revised 2017 Ethiopian Federal Ministry of Health HIV Care/ART Follow-Up Form, which was used in the ART clinic and by using different peer-reviewed published literature [6-8, 16-20]. Secondary data from three public health facilities of Shashamene town were used to retrieve data from the initial date of ART up to the end of the follow-up (from January 1, 2011, to December 30, 2015, and followed up until February 30, 2018). All HIV-positive children on care and support follow-up who had started ART at public health facilities of Shashamene town and fulfilled the inclusion criteria were included in the study.

2.7. Data Quality Assurance. The quality of the data was assured by caring out a careful design of the checklist. Training was given for data collectors and supervisors and a pretest was conducted before data collection. Finally, five percent of the completed data was selected randomly and cross-checked with medical cards of the patients to check for consistency at the end of each day. The collected data were checked for its completeness manually. Data coding and entry was done using EpiData version 3.1.

2.8. Data Analysis Procedure. The data were exported from EpiData to SPSS version 20 for data analysis. Data organizing was done to transform the data into a format suitable for further analysis. Finally, two standard statistical methods were employed: a nonparametric method called the Kaplan-Meier method and the associated log-rank test, and a semiparametric method known as the Cox proportional hazards regression model. The Kaplan-Meier method was used to estimate the survival probability after the initiation of ART to first-line treatment failure, and a log-rank test was 
used to compare survival curves of children between different categories of predictor variables at $p$ value $(<0.05)$.

The Cox proportional hazard regression model was used to determine the predictors of time to first-line treatment failure. In the bivariable model, covariables with $p$ values $(<0.25)$ were selected to be included in the multivariable model. The interaction effect was assessed for possible covariables at $p$ value of 0.05 , and the model fitness was checked using log minus log-survival curve for predictor variables. The final model was interpreted using the adjusted hazard ratio (AHR) with 95\% confidence interval and $p$ value $<0.05$ to measure the predictors of the first-line treatment failure.

2.9. Ethical Consideration. Ethical clearance was obtained from the Institutional Review Board (IRB) of Institute of Health, Jimma University. In addition, permission letter was sought from West Arsi Zonal Health Office and each health facility administrative. The retrieved data were kept strictly confidential and the names of children or their parents were not included.

\section{Results}

3.1. Sociodemographic Characteristics. A total of 484 medical cards of children living with HIV/AIDS and who started ART drugs were reviewed, of which 70 (14.5\%) were excluded following the exclusion criteria. Finally, a total of 414 children were included in the analysis. Half $(212(51.2 \%))$ of the children were male. Majority of them 268 (64.7\%) were above 5 years at the initiation of ART. The mean age of children at the initiation of ART was 6.6 years with $(\mathrm{SD} \pm 3.8)$ (Table 1).

3.1.1. Baseline Clinical Status of the Study Subjects. Among $414 \mathrm{HIV}$-positive children enrolled on ART, 383 $(92.5 \%)$ and $202(48.8 \%)$ of them received cotrimoxazole and isoniazide prophylaxis initiation, respectively. Of the study participants, 85 (20.5\%) were anemic and 128 (30.9\%) had CD4 count below the threshold level during ART initiation (Table 2).

3.1.2. Outcome and Survival Status of Children. From a total of children initiated HAART during the study period, 27 $(6.5 \%)$ of them had virological failure, $17(4.1 \%)$ had immunological failure, 20 (4.8\%) had clinical failure, 37 (8.9\%) died, 47 (11.4\%) were lost to follow-up, 44 (10.6\%) were transferred out, and $222(55.6 \%)$ were active. The median follow-up time was 38 months with interquartile range (IQR) of 24 to 52 months, and the median survival time to virological failure was 30 months with IQR of 24.4 to 44.2.

The cumulative probability of survival status of children after $1^{\text {st }}, 2^{\text {nd }}, 3^{\text {rd }}$, and $4^{\text {th }}$ years was $81 \%, 41 \%, 19 \%$, and $4 \%$, respectively (Figure 1 ). This indicates that as the child received ART for a long period of time, the probability of treatment failure increases. The incidence density of
TABLE 1: Sociodemographic characteristics of children who started ART at public health facilities between January 1, 2011, to December 30, 2015, in Shashamene town, Oromia Region, Ethiopia, 2019.

\begin{tabular}{lccc}
\hline Variables & Category & No. & $(\%)$ \\
\hline \multirow{2}{*}{ Sex } & Female & 202 & 48.8 \\
& Male & 212 & 51.2 \\
\hline \multirow{3}{*}{ Age } & $<1$ year & 27 & 6.5 \\
& $1-5$ years & 119 & 28.7 \\
& $\geq 5$ years & 268 & 64.7 \\
\multirow{2}{*}{ Child's caregiver } & Parents & 335 & 80.9 \\
& Sibling & 64 & 15.5 \\
& Orphan & 13 & 3.1 \\
\multirow{4}{*}{ Nutritional history } & Exclusive breastfeeding & 147 & 35.5 \\
& No exclusive breastfeeding & 261 & 63 \\
& Appropriate for age & 312 & 75.9 \\
\multirow{2}{*}{ Developmental milestone } & Delayed & 76 & 18.5 \\
& Regressed & 23 & 5.6 \\
\hline \multirow{3}{*}{ Immunization status } & Appropriate for age & 218 & 52.7 \\
& Not appropriate for age & 140 & 33.8 \\
& Not immunized & 54 & 13 \\
\hline
\end{tabular}

TABLE 2: Baseline clinical status among children who started ART at public health facilities between January 1, 2011, and December 30, 2015, in Shashamene town, Oromia Region, Ethiopia, 2019.

\begin{tabular}{|c|c|c|c|}
\hline Variables & Category & No. & $(\%)$ \\
\hline \multirow{2}{*}{$\begin{array}{l}\text { Cotrimoxazole prophylaxis } \\
\text { initiation }\end{array}$} & Yes & 383 & 92.5 \\
\hline & No & 31 & 7.5 \\
\hline \multirow{2}{*}{ Isoniazide prophylaxis initiation } & Yes & 203 & 49 \\
\hline & No & 211 & 51 \\
\hline \multirow{2}{*}{ Baseline Hgb status } & Anemic & 85 & 20.5 \\
\hline & Normal & 319 & 77.1 \\
\hline \multirow{2}{*}{$\begin{array}{l}\text { TB smear test result during } \\
\text { follow-up }\end{array}$} & Positive & 90 & 21.7 \\
\hline & Negative & 303 & 73.2 \\
\hline \multirow[t]{2}{*}{ Baseline CD4 count } & $\begin{array}{c}\text { Below threshold } \\
\text { level }\end{array}$ & 128 & 30.9 \\
\hline & Normal & 286 & 69.1 \\
\hline \multirow{2}{*}{ Baseline WHO stage } & Stages 1 and 2 & 271 & 65.5 \\
\hline & Stages 3 and 4 & 143 & 34.5 \\
\hline \multirow{2}{*}{ Baseline nutritional status } & Normal & 217 & 52.4 \\
\hline & Undernutrition & 197 & 47.7 \\
\hline \multirow{2}{*}{ Exposure status for PMTCT } & Exposed & 132 & 31.9 \\
\hline & Not exposed & 197 & 47.6 \\
\hline \multirow{2}{*}{ Regime } & Nonnevirapine & 172 & 41.5 \\
\hline & Nevirapine & 240 & 58 \\
\hline \multirow{3}{*}{ Adherence } & Good & 251 & 60.6 \\
\hline & Fair & 82 & 19.8 \\
\hline & Poor & 80 & 19.3 \\
\hline
\end{tabular}

treatment failure was 16 children per 10,000 person months with a total of 16,583 person months of follow-up.

3.1.3. Comparison of Survival Functions. Children in WHO clinical stages one and two survived significantly longer than those in clinical stages three and four after HAAR initiation before treatment failure $(\log$-rank, $p$ value $=0.001)$. Children 


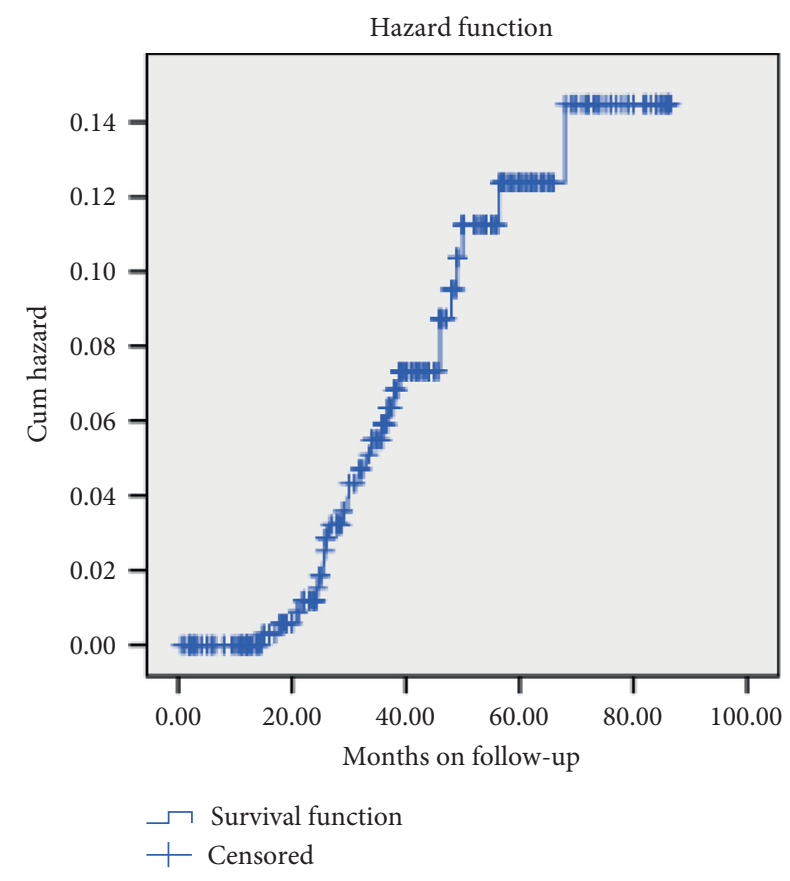

FIgURE 1: Cumulative probability of first-line ART failure among patients in Shashamene town health facilities.

with CD4 count above the threshold level at baseline survived more before treatment failure compared to their counterparts. This difference was also statistically significant $(\log$-rank, $p$ value $=0.003)($ Figures 2 and 3$)$.

\subsubsection{Sociodemographic Predictors of Time to Treatment} Failure among Children Started ART. From sociodemographic factors during bivariable Cox regression, only sex being male was nominated for multivariable analysis (Table 3).

\subsection{Bivariable Cox Regression Analysis for Clinical Predictors.} From clinical factors during bivariable Cox regression, having anemia during the start of ART, TB infection during the follow-up, baseline advanced WHO clinical stages (stages 3 and 4), baseline CD4 count below threshold level, and usage of nevirapine at baseline were nominated for multivariable Cox regression analysis (Table 4).

\subsubsection{The Overall Predictors of Time to First-Line Treatment} Failures among HIV-Infected Children on ART. From the multivariable Cox regression analysis, children in WHO stages 3 and 4 during the initiation of ART were 5.7 times at higher risk of treatment failure $(\mathrm{AHR}=5.69(95 \% \mathrm{CI}$ : 2.07-15.66) compared to children in WHO stages 1 and 2; and children treated with NVP-based NNRT initially were

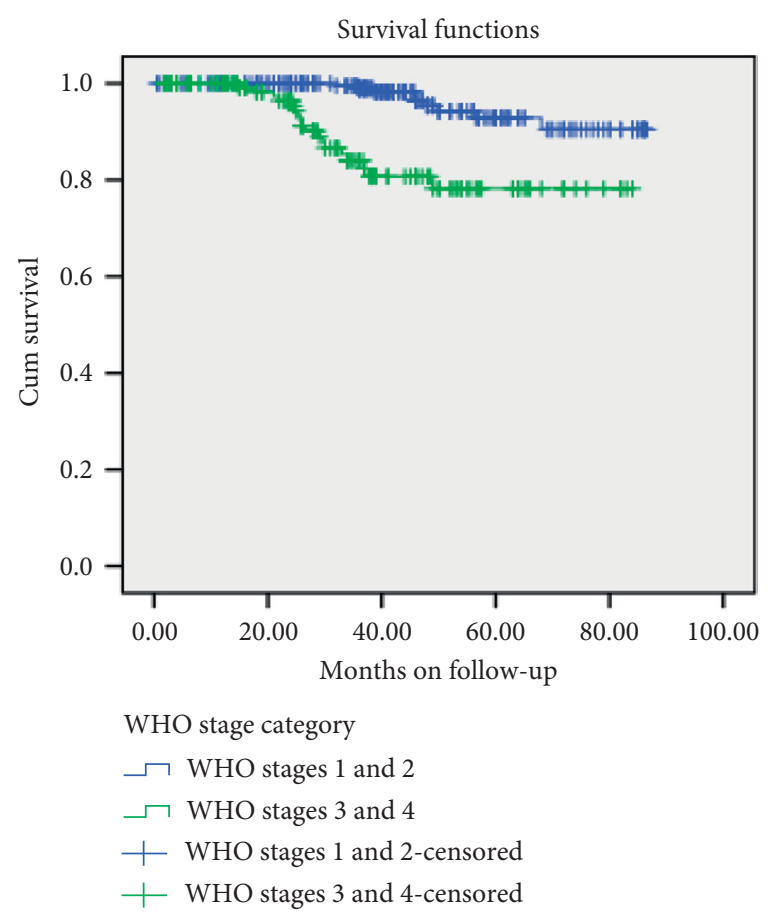

FIgURE 2: Survival curves for the cohort of children on ART according to their WHO clinical stage.

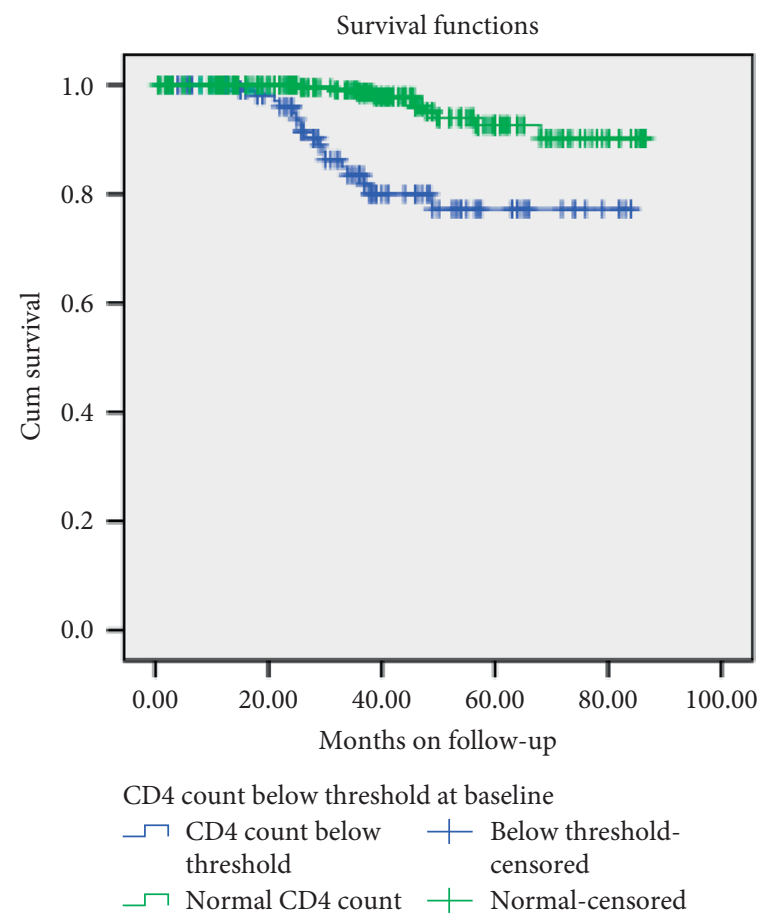

Figure 3: Survival curves for the cohort of children on ART according to their CD4 count. 
TABLE 3: Bivariable Cox regression analysis for sociodemographic determinants of survival among children started ART at public health facilities between January 1, 2011, and December 30, 2015, at Shashamene town, Oromia Region, Ethiopia.

\begin{tabular}{|c|c|c|c|c|}
\hline Variables & Category & No. (\%) & CHR $(95 \% \mathrm{CI})$ & $p$ value \\
\hline \multirow{2}{*}{ Sex } & Female & $202(48.8)$ & 1 & \\
\hline & Male & $212(51.2)$ & $1.72(0.727-4.05)$ & 0.21 \\
\hline \multirow{3}{*}{ Age } & $<1$ year & $27(6.5)$ & $0.305(0.39-2.421)$ & 0.26 \\
\hline & $1-5$ years & $119(28.7)$ & $1.311(0.548-3.13)$ & 0.54 \\
\hline & $\geq 5$ years & $268(64.7)$ & 1 & \\
\hline \multirow{3}{*}{ Child's caregiver } & Parents & $335(80.9)$ & 1 & \\
\hline & Sibling & $64(15.5)$ & $1.35(0.49-3.68)$ & 0.55 \\
\hline & Orphan & $13(3.1)$ & & \\
\hline \multirow{3}{*}{ Nutritional history } & Exclusive breastfeeding & $147(35.5)$ & 1 & \\
\hline & No exclusive breastfeeding & $261(63)$ & $0.93(0.37-2.75)$ & 0.9 \\
\hline & Appropriate for age & $312(75.9)$ & 1 & \\
\hline \multirow{2}{*}{ Developmental milestone } & Delayed & $76(18.5)$ & $1.24(0.50-3.06)$ & 0.64 \\
\hline & Regressed & $23(5.6)$ & $2(0.56-7.14)$ & 0.28 \\
\hline \multirow{3}{*}{ Immunization status } & Appropriate for age & $218(52.7)$ & 1 & \\
\hline & Not appropriate for age & $140(33.8)$ & $1.0(0.39-2.56)$ & 0.99 \\
\hline & Not immunized & $54(13)$ & $0.78(0.28-2.21)$ & 0.65 \\
\hline
\end{tabular}

TABLE 4: Bivariable Cox regression analysis for clinical determinants of survival among children who started ART at public health facilities between January 1, 2011, to December 30, 2015, at Shashamene town, Oromia region, Ethiopia.

\begin{tabular}{|c|c|c|c|c|}
\hline Variables & Category & No. $(\%)$ & CHR $(95 \% \mathrm{CI})$ & $p$ value \\
\hline \multirow{2}{*}{ Cotrimoxazole prophylaxis initiation } & Yes & $383(92.5)$ & 1 & \\
\hline & No & $31(7.5)$ & $0.96(0.39-7.27)$ & 0.97 \\
\hline \multirow{2}{*}{ Isoniazide prophylaxis initiation } & Yes & $202(48.8)$ & 1 & \\
\hline & No & $211(51)$ & $1.53(.59-3.92)$ & 0.37 \\
\hline \multirow{2}{*}{ Baseline Hgb status } & Anemia & $85(20.5)$ & $3.87(1.42-10.57)$ & 0.01 \\
\hline & Normal & $319(77.1)$ & 1 & \\
\hline \multirow{2}{*}{ TB result } & Positive & $90(21.7)$ & $2.18(0.89-5.36)$ & 0.08 \\
\hline & Negative & $303(73.2)$ & 1 & \\
\hline \multirow{2}{*}{ Baseline CD4 count } & Below threshold level & $128(30.9)$ & $4.37(1.37-11.14)$ & 0.002 \\
\hline & Normal & $286(69.1)$ & 1 & \\
\hline \multirow{2}{*}{ Baseline WHO stage } & Nonadvanced stage (stages 1 and 2 & $271(65.5)$ & 1 & \\
\hline & Advanced stage (stages 3 and 4 ) & $143(34.5)$ & $5.35(2.0-14.30)$ & 0.001 \\
\hline \multirow{4}{*}{ Baseline nutritional status } & $Z$ score $>-2$ & $143(34.5)$ & 1 & \\
\hline & $\mathrm{Z}$ score $\leq-2$ & $24(5.8)$ & $1.43(.28-7.84)$ & 0.66 \\
\hline & $\mathrm{BMI}<16 \mathrm{~kg} / \mathrm{m}^{2}$ & $271(65.5)$ & $1.59(0.64-4.07)$ & 0.33 \\
\hline & $\mathrm{BMI} \geq 16 \mathrm{~kg} / \mathrm{m}^{2}$ & $143(34.5)$ & 1 & \\
\hline \multirow{2}{*}{ Exposure status for PMTCT } & Exposed & $132(31.9)$ & $1.23(0.48-3.15)$ & 0.66 \\
\hline & Not exposed & $197(47.6)$ & 1 & \\
\hline \multirow{2}{*}{ Regime } & Nonnevirapine & $172(41.5)$ & 1 & \\
\hline & Nevirapine & $240(58.0)$ & $2.43(1.04-5.67)$ & 0.04 \\
\hline \multirow{3}{*}{ Adherence } & Good & $251(60.6)$ & 1 & \\
\hline & Fair & $82(19.8)$ & $0.646(0.13-3.29)$ & 0.6 \\
\hline & Poor & $81(19.6)$ & $1.349(0.39-4.61)$ & 0.63 \\
\hline
\end{tabular}

2.7 times at higher risk of treatment failure $(\mathrm{AHR}=2.72$ (1.13-6.54)) compared to children treated with non-NVP (Table 5).

\section{Discussion}

The study has provided pertinent information about predictors with time to treatment failure among children living with HIV enrolled on ART which can support activities being implemented to prevent the occurrence of early firstline treatment failure for planners and decision makers. From a total of children initiated HAART during the study period, $27(6.5 \%)$ of them had virological failure with the median survival time of 30 months with an IQR of 24.42 to 44.25. This time was similar to that reported in the study done in South London; the median duration of antiretroviral therapy at treatment failure was 30 months (range: 13 months -5 years) [21] but it was high as compared to the 
TABLE 5: The overall predictors of time to first-line treatment failure among children started ART.

\begin{tabular}{|c|c|c|c|}
\hline Variables & Category & AHR $(95 \%$ CI $)$ & $p$ value \\
\hline \multirow{2}{*}{ Sex } & Male & 1 & \\
\hline & Female & $1.07(0.37-3.02)$ & 0.9 \\
\hline \multirow{2}{*}{ Hgb level } & Anemia & $2.28(0.77-6.78)$ & 0.14 \\
\hline & No anemia & 1 & \\
\hline \multirow{2}{*}{$\mathrm{TB}$ result } & Positive & $1.30(0.45-3.71)$ & 0.63 \\
\hline & Negative & & \\
\hline Baseline CD4 count & $\begin{array}{c}\text { Below threshold level } \\
\text { Normal }\end{array}$ & $1.13(0.29-9.96)$ & 0.91 \\
\hline \multirow{2}{*}{ Baseline WHO stage } & Stages 1 and 2 & 1 & \\
\hline & Stages 3 and 4 & $5.69(2.07-15.66)$ & 0.001 \\
\hline \multirow{2}{*}{ Treatment regime } & Nonnevirapine & 1 & \\
\hline & Nevirapine & $2.72(1.13-6.54)$ & 0.02 \\
\hline
\end{tabular}

study done in Uganda and Mozambique [6]. This difference could be due to the difference in follow-up period and the study settings.

The incidence density of the treatment failure in this study was 16 children per 10,000 person months with a total of 16,583 person months of follow-up. This result was low as compared to the study done in Amhara Regional State, which was 22.1 per 10,000 person months [22]. The reason for the lowest incidence density could be related to the difference in the study period/setting and this finding focused only on virological failure.

Children in the advanced WHO clinical stage (stages 3 and 4) during the initiation of ART were 5.7 times at higher risk of treatment failure than their counterparts at $\mathrm{WHO}$ stages 1 and 2, which is consistent with the study done in Mozambique and Uganda; children in WHO stages 3 and 4 were significantly more likely to experience treatment failure [6]. This could be due to the fact that children in advanced WHO clinical stages are more likely to have advanced immune suppression and higher rate of comorbidities so that the risk of ART failure could be higher.

Children treated with NVP-based NNRT initially were 2.7 times at higher risk of treatment failure compared to their counterparts. This result was in line with the study done in South Africa, where the use of nevirapine-based regime in the initial stage increases the risk for early treatment failure [23]. The study done in Uganda and Tanzania on predictors of virological failure also supports this finding [24, 25]. This could be due to low dose of NVP drug transmission to infants through placenta or breast feeding during PMTCT.

The strength and limitations of this study are as follows:

Strength: the study uses a strong design and a longer follow-up duration to estimate the survival time and independent predictors of first-line ART failure

Limitation: restriction of incomplete data may underestimate or overestimate this result; there are limited data on key possible predictors of VF such as tuberculosis coinfection and PMTCT. Missing data on variables may limit the range of variables and the number of children that could be included in multivariable models.

\section{Conclusion and Recommendation}

The median survival time of first-line antiretroviral treatment failure was moderate in the study area as compared to other studies. The finding also demonstrated that children treated with NVP-based NNRT at the initial and advanced WHO clinical stages at baseline were at higher risk of treatment failure. Therefore, Ministry of Health, stakeholders, and healthcare providers should give attention to ensure early diagnosis and enrollment into ART. Health workers need to give more emphasis during clinical care for patients taking NVP-based NNRT treatment and who are in advanced WHO clinical stages. The finding of increased rates of virological failure among children receiving nevirapine suggests that more work should be done by the Ministry of Health to make efavirenz a cost-effective option for pediatric antiretroviral treatment programs in the country.

\section{Abbreviations}

AHR: $\quad$ Adjusted hazard ratio

ART: Antiretroviral therapy

ARTF: Antiretroviral therapy failure

BMI: $\quad$ Body mass index

CHR: $\quad$ Crude hazard ratio

cART: Combination antiretroviral therapy

EPHIC: Ethiopian Pediatric HIV Cohort

HAART: Highly active antiretroviral therapy

HIV/AIDS: Human immunodeficiency virus/acquired immunodeficiency syndrome

IQR: Interquartile range

LMIC: $\quad$ Low-middle-income countries

NNRT: Nonnucleotide reverse transcriptase

NVP: $\quad$ Nevirapine

OI: Opportunistic illness

PLWH: People living with HIV 
PMTCT: Prevention of mother-to-child transmission WHO: World Health Organization.

\section{Data Availability}

The raw datasets used and/analyzed during the current study are available from the corresponding author on reasonable request.

\section{Ethical Approval}

Ethical clearance was obtained from the Institutional Review Board (IRB) of Institute of Health, Jimma University.

\section{Consent}

Permission letter was sought from Oromia Regional Health Bureau for each health facility. The retrieved data were kept strictly confidential and the names of children or their parents were not included.

\section{Conflicts of Interest}

The authors have no conflicts of interest.

\section{Authors' Contributions}

EZ participated in data collection, participated in data collection, conducted the data analysis and interpretation, and developed the first draft. AW contributed to study conception, data analysis, and interpretation of data and revised subsequent drafts. AA reviewed and commented on successive drafts. All authors read and approved the final manuscript.

\section{Acknowledgments}

The authors would like to thank Mr. Henok Assefa and Mr. Solomon Berhanu for their support and guidance for the development of this research study. They want to sincerely acknowledge data collectors, supervisors, and those who are working in Abosto Health Center, Shashemene referral hospital, and Melka Oda General Hospital for their cooperation.

\section{References}

[1] E. Ek and World Health Organization, Taking Stock: HIV in Children: The State of Affairs, World Health Organization, Geneva, Switzerland, 2006.

[2] U. C. Agozi, "Pediatric HIV/AIDS in sub-Saharan Africa: emerging issues and wayforward," African Health Sciences, vol. 12, no. 3, pp. 297-304, 2012.

[3] "Panel on antiretroviral therapy and medical management of children living with HIV. guidelines for the use of antiretroviral agents in pediatric HIV infection," 2016, http:// aidsinfo.nih.gov/content-\%20files/lv\%20guidelines/pediatric guidelines.pdf.

[4] World Health Organization Report, ART Failure and Strategies for Switching ART Regimens in the WHO European Region, WHO, Geneva Switzerland, 2007.
[5] A. Mwangi, C. M. Kwobah, A. W. Mwangi, J. K. Koech, G. N. Simiyu, and A. M. Siika, "Factors associated with firstline antiretroviral therapy failure amongst HIV-infected African patients: a case-control," BMC Infectious Disease, vol. 19, p. 537, 2014.

[6] P. Costenaro, M. Penazzato, R. Lundin et al., "Predictors of treatment failure in HIV-positive children receiving combination antiretroviral therapy: cohort data from Mozambique and Uganda," Journal of the Pediatric Infectious Diseases Society, vol. 4, no. 1, pp. 39-48, 2018.

[7] H. Barennes, K. Virak, F. Rouet, Y. Buisson, M. Strobel, and U. Vibol, "Factors associated with the failure of first and second-line antiretroviral therapies therapy, a case control study in Cambodian HIV-1 infected children," BMC Research Notes, vol. 9, pp. 1-10, 2016.

[8] B. T. Tadesse, B. A. Foster, D. Jerene, and A. Ruff, "Cohort pro fi le : improving treatment of HIV-infected Ethiopian children through better detection of treatment failure in southern Ethiopia," BMJ Open, vol. 7, no. 2, Article ID e013528, 2017.

[9] Y. A. Melsew, M. W. Terefe, G. A. Tessema, and T. A. Ayele, "AIDS \& clinical rate of immunological failure and its predictors among patients on highly active antiretroviral therapy at debremarkos hospital, Northwest Ethiopia : a retrospective follow up study," Journal of AIDS \& Clinical Research, vol. 4, no. $5,2013$.

[10] A. Plan and H. I. V. D. Resistance, Global Action Plan on HIV Drug Resistance 2017-2021, WHO, Geneva, Switzerland, 2017.

[11] J. Mullen, S. Leech, S. O. Shea et al., "Antiretroviral drug resistance among HIV-1 infected children failing treatment," Journal of Medical Virology, vol. 68, no. 3, pp. 299-304, 2002.

[12] S. Desmonde, K. Malateste, F. Dicko et al., "Determinants of durability of first-line antiretroviral therapy regimen and time from first-line failure to second-line antiretroviral therapy initiation Sophie," AIDS (London, England), vol. 29, no. 12, pp. 1527-1536, 2018.

[13] World Health Organization Report, Case Definitions of HIV for Surveillance and Revised Clinical Staging and Immunological Classification of HIV-Related Disease, WHO, Geneva, Switzerland, 2006.

[14] World Health Organization, Haemoglobin Concentrations for the Diagnosis of Anaemia and Assessment of Severity, WHO, Geneva, Switzerland, 2011.

[15] Federal Ministry of Health, Health of. National Guidelines for Comprehensive HIV Prevention, Care and Treatment, Federal Ministry of Health, Abuja, Nigeria, 2017.

[16] A. Zeleke, "Prevalence of antiretroviral treatment failure and associated factors in HIV infected children on antiretroviral therapy at Gondar University hospital, retrospective cohort study," International Journal of Medicine and Medical Sciences, vol. 8, no. 11, pp. 125-132, 2016.

[17] B. P. Westley, A. K. Delong, C. S. Tray et al., "Prediction of treatment failure using 2010 World health organization guidelines is associated with high misclassification rates and drug resistance among HIV-infected Cambodian children," Clinical Infectious Diseases, vol. 55, no. 3, pp. 432-430, 2017.

[18] C. M. Kwobah, A. Mwangi, J. Koech, G. N. Simiyu, and A. M. Siika, "Factors associated with first-line antiretroviral therapy failure amongst HIV-infected african patients: a casecontrol study," World Journal of AIDS, vol. 2, no. 4, pp. 271-278, 2012.

[19] K. Jobanputra, L. A. Parker, C. Azih et al., "Factors associated with virological failure and suppression after enhanced adherence counselling, in children, adolescents and adults on 
antiretroviral therapy for HIV in Swaziland," PLoS One, vol. 450, pp. 1-12, 2015.

[20] T. Bacha, B. Tilahun, and A. Worku, "Predictors of treatment failure and time to detection and switching in HIV-infected Ethiopian children receiving first line anti-retroviral therapy," BMC Infectious Disease, vol. 12, p. 197, 2012.

[21] S. Hussain, A. Hajjar, H. Frayha, and S. Althawadi, vol. 2012, pp. 565-9 original article.

[22] M. M. Sisay, T. A. Ayele, Y. A. Gelaw, A. T. Tsegaye, K. A. Gelaye, and M. F. Melak, "Incidence and risk factors of first-line antiretroviral treatment failure among human immunodeficiency virus- infected children in Amhara regional state, Ethiopia : a retrospective follow-up study," BMJ Open, vol. 8, no. 4, Article ID e019181, 2018.

[23] M.-A. Davies, H. Moultrie, B. Eley et al., "Virologic failure and second-line antiretroviral therapy in children in South Africathe IeDEA Southern Africa collaboration," JAIDS Journal of Acquired Immune Deficiency Syndromes, vol. 56, no. 3, pp. $270-278,2011$.

[24] M. R. Kamya, H. Mayanja-Kizza, A. Kambugu et al., "Predictors of long-term viral failure among Ugandan children and adults treated with antiretroviral therapy," JAIDS Journal of Acquired Immune Deficiency Syndromes, vol. 46, no. 2, pp. 187-193, 2007.

[25] S. D. Emmett, C. K. Cunningham, B. T. Mmbaga et al., Study, vol. 54, no. 4 , pp. $368-375,2014$. 